\title{
Research on Preparation Technology of Silicide Thermoelectric Material and Its Thermoelectric Transport Properties
}

\author{
Shiyun Zhou \\ School of Electronic and Information Engineering, Anshun University, Anshun, 561000, China
}

Keywords: Silicide thermoelectric material, Preparation technology, Thermoelectric transport properties

\begin{abstract}
Thermoelectric material is a kind of carrier material which will be able to use in the conversion of new functional electrical and thermal energy. It has the advantages of light weight, long life, small size, clean, environmental protection and many other advantages, which has been popularized in the automobile exhaust gas recycling and industrial waste heat. There are many kinds of thermoelectric materials, among which magnesium silicon materials have attracted extensive attention due to the rich raw materials, non-toxic, safe and reliable characteristics. In this paper, the preparation technology and thermoelectric transmission properties of silicide thermoelectric materials are analyzed to provide some references for the relative researchers.
\end{abstract}

\section{Introduction}

Thermoelectric material is a new type of functional material which can convert the electric energy and thermal energy directly by the carrier movement in the material. This material has many advantages, such as light weight, long life, small volume, no danger, clean, environmental protection and so on, and can be used as the key development of green materials. At present, thermoelectric materials have been widely used in aerospace, aviation, military and other highly sophisticated fields, and have been initially promoted in the recovery of automobile exhaust and industrial waste heat and waste heat recycling. In the past, the low conversion efficiency and high production cost of thermoelectric materials become the main factors restricting the development of thermoelectric materials. Thermoelectric conversion materials have great potential in the utilization of waste heat power generation, which is of great significance for solving the energy problems and environmental problems, and building a low carbon society. Therefore, the research of thermoelectric materials and their related energy conversion technology has become a rapidly growing and active research field in the world in recent years. Japan, the United States, Europe and other countries and regions have carried out a lot of research in this field. In our country, the research and application of thermoelectric materials technology has been national attention and support, has started the thermoelectric conversion materials and devices related countries and projects, and has been included in the focus of the National Natural Science Foundation of China, which effectively promoted the development of China's thermal power field. Believe that through unremitting efforts, thermoelectric technology will be more competitive in the power generation and refrigeration. As a kind of environmental friendly thermoelectric material, metal silicide thermoelectric materials have many advantages: the abundance of elements in the crust is high, the price is low, the oxidation resistance is good at high temperature, and the chemical properties are stable. Since the study of thermoelectric properties of metal silicide thermoelectric materials, metal silicide materials have attracted more and more attention of thermoelectric materials researchers. 


\section{Preparation Technology of Silicide Thermoelectric Material}

\subsection{Experimental Materials and Instruments}

Mn powder (99.9\%, Alfa, Aesar) Si powder (99.9\%, Alfa, Aesar) VGB type vacuum glove box (Anhui Beiyike), W2V20 (Korea uzin vacuum mechanical pump), FA1004 type electronic balance (Shanghai flat) and HY-SG1500 type microwave tube furnace (China Metallurgical microwave) STX-202 type diamond wire cutting machine (Shenyang crystal), UNIPOL-1202 polishing machine, Rigaku-Mini type Felex600 X ray diffraction ZEISS-ULTRA55 scanning electron microscope (SEM); thermoelectric parameters testing system of Namiceo (Wuhan Jia Yi Tong). Two high strength ultra-low humidity electronic industry level ten dry cabinet; digital electronic balance, a sense of the amount of 0.1M; MFAS-25 type induction furnace DEGUSSA; 5kW high temperature box furnace, MTI GSL1600x40 vacuum tube high temperature furnace; MTI OTF-1200X, a 80SL open type annealing furnace; Dr.SinterSPS.1050 spark plasma sintering furnace in Japan Sumitomo stone mining Corporation; ultra-high frequency heating or melting furnace; HVDS-II high vacuum spinning machine (molecular pump vacuum system); vacuum hot press is designed, (equipped with a microcomputer controlled electro-hydraulic servo pressure testing machine, vacuum pump system, high purity graphite mold).

\subsection{Preparation Process of High Manganese Silicon}

To study the effect of substitution and rapid solidification grain refining on the thermoelectric properties of high manganese silicon materials. The high manganese silicon ingot was obtained by levitation melting and melted under the protection of hydrogen. The peak power of the experiment was as follows. To compare the effect of rapid solidification on the properties of the material, a thin sheet of rapidly solidified alloy was prepared by means of tape casting. The melted sample and the rejected sample are grinded and sieved to the target. The powder is sintered by spark plasma sintering, and the sintering parameters are fine. The final sample is a disk-shaped alloy with a diameter of one thickness. Compared with the melting method, the solid phase reaction has the advantages of low reaction temperature and easier control of volatilization. In view of the above analysis, this study adopts the method of solid phase reaction by silicification from Keng Keng, donor, preparation of doped materials. Magnesium powder, silicon powder and silicon powder in the glove box from Keng, weighing and mixing under pressure cold forming of tube furnace through hydrogen flow insulation get loose powder blue, pure phase under test, vacuum hot pressing sample, vacuum hot pressing equipment photo map is designed by research team, equipped with diffusion the vacuum pump system, using high pure graphite mould, vacuum hot pressing technology is a powder sintering, after grinding powder solid phase reaction to sieve mesh in graphite mold, the mold is arranged on the machine in the sample table, lifting sample table by the micro machine control electro-hydraulic servo pressure testing machine to control. The pure Mn (> 99.7\%) and high purity (Si > 99.999\%) as raw material, industrial pure manganese by nitric acid alcohol solution to remove the oxide layer after pickling, according to MnSix (x=1.60, 1.65, 1.68, 1.73, 1.81, 1.85) stoichiometric ingredients, the raw materials are put into a high frequency magnetic induction melting in argon gas the furnace smelting ingot ingot will get 3 4 times, sealed quartz tube and vacuum at 850 DEG C, homogenizing annealing $8 \mathrm{~h}$, then annealed samples by $1 \mathrm{H}$ milling and sieving. The strength of the electron phonon interaction effect on thermoelectric conversion by analyzing the phonon spectrum, the optical phonon contribution of high frequency electro acoustic the son was. By comparing the phonon density and the spectral function, it is found that the vibration of the atom has a significant influence on the electron phonon interaction. The phonon properties and thermodynamic properties of solid solutions were investigated by density functional perturbation theory. It is found that with the increase of the content, the phonon group velocity decreases and the phonon thermal conductivity is reduced. This is mainly due to the large mass of atoms and the weakening of chemical bonds at the same time. Finally, the sample powder in graphite mold, HMS block sample and thermal insulation $5 \mathrm{~min}$ in vacuum, 850 
DEG C, 30MPa SPS under the condition of sintering was $20 \mathrm{~mm}$ in diameter and $4 \mathrm{~mm}$. Using the RIGAKU D/max-3c type X X-ray diffractometry (CuK alpha, lambda $=0.154059 \mathrm{~nm}$ ) on sintering samples phase analysis, surface the morphology of sintered samples was observed by FEINanoSEM scanning electron microscope, the Seebeck coefficients of the samples and the resistivity using standard four probe methods (ULVACZEM-2) were tested by laser thermal conductivity perturbation method (ULVACTC-7000) test.

\subsection{Characterization of Samples}

XRD powder diffraction patterns of high manganese silicon (HMS) samples synthesized by microwave sintering at 900 degrees $C$ respectively represent $S i$ excess of $7 \%$, 6\%, 5\% mole in raw powder, and three XRD diffraction patterns of different samples are prepared. According to Figure 3-1, the main phases of the three samples are all high manganese silicon phases, and the group space is $\mathrm{p}-4 \mathrm{n} 2$. Through the contrastive analysis, when the silicon more than $5 \%$ Moore, MnSi wave are sintered samples, but with the increase of silicon content, the diffraction peak of MnSi diffraction peaks to disappear, silicon, silicon more than 5\% when Moore, just get samples for high manganese silicon (HMS) pure phase. The results are in good agreement with the relevant reports. The synthesis of high manganese silicon is divided into two processes, $\mathrm{Mn}+\mathrm{Si}, \mathrm{MnSi}$ and $\mathrm{MnSi}+0.74 \mathrm{Si}$ to MnSi1.74, while the increase of Si content is beneficial to the positive reaction and inhibits the formation of MnSi.

\section{Thermoelectric Transport Properties of Silicide Thermoelectric Material}

$\mathrm{Mg}_{2} \mathrm{Si}$ has an anti-fluorite structure with a space group of $3 \mathrm{M}$, and the structure of the sample is shown in figure 1 . Si is located at the face center and the eight vertex angles of the unit cell, and the $\mathrm{Mg}$ atom lies at the center of the 8 small cubes, located at 025ao, 0.25, 0.25. A $\mathrm{Mg}_{2} \mathrm{Si}$ cell model is used in the calculation, which consists of $8 \mathrm{Mg}$ primitive and $4 \mathrm{Si}$ primitive. The Wien2K software package based on the FLAPW method is adopted and the Perdew-Burke-Ernzerhof (PBE) exchange correlation potential under the generalized gradient approximation (GGA) is used to solve the Kohn-Sham equation. The value of the convergence parameter fox is set to 8 and 0 , in which the fire MT is the cake radius, and the Kmax is the largest inverted vector in the plane wave expansion. The calculated values of MT for both $\mathrm{Mg}$ and $\mathrm{Si}$ are $0.13 \mathrm{~nm}$, and the energy convergence criterion for self-consistent calculation is 1 x 10.5Ry. The crystal structure of $\mathrm{Mg}_{2} \mathrm{Si}$ is shown in Figure 1.

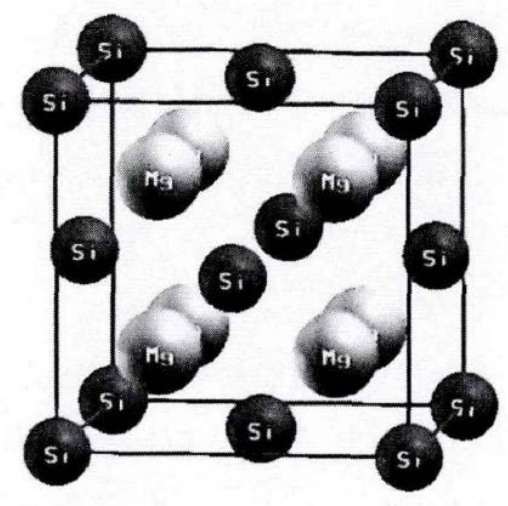

Figure 1. Crystal structure of $\mathrm{Mg}_{2} \mathrm{Si}$

In the structural optimization and autonomous calculation, Brillouin region $10 * 10 * 10$ grid point is adopted. The thermoelectric transport coefficient has been calculated by the semiclassical Boltzmann transport equation and the rigid band approximation (RBA). This method has been successfully applied to the transport properties of several thermoelectric materials. Because of the Brillouin zone grid points on transport properties calculation of shadow throat larger, to improve the transmission accuracy properties, increase the number of k points in the calculation of $24000 \mathrm{~K}$ point in the Brillouin zone eigenvalue calculation. When the structure is optimized, the energy of the 
system varies with the volume, as shown in figure 3.2. We can see through the GGA optimization system in steady state Mg2Si cell volume is 437.0874 a.u.3, where $1 \mathrm{a} . \mathrm{u} .=0.0529 \mathrm{~nm}$, the lattice constant of $\mathrm{Mg} 2 \mathrm{Si}$ is $0.6373 \mathrm{~nm}$, the calculated results and the experimental value of $0.6338 \mathrm{~nm}$ is basically the same, indicating that the calculation parameter selection is appropriate. On this basis, the GGA method is used to calculate the electronic structure of the stable lattice structure. The band structure of Mg2Si in the symmetry direction along Brillouin zone is calculated. As shown in Figure 2, the dotted line is the position of Fermi energy level. The Fermi level is chosen as the energy zero point, and the other energy is the relative value of the Fermi level as the reference standard. It can be seen from the energy band that the valence band top and conduction band bottom are located at $(0,0$, $0)$ and $X(0,0,0.5)$ points, respectively, indicating that Mg2Si is an indirect band gap semiconductor. The band gap is $\mathrm{E}(\mathrm{F})-\mathrm{E}(\mathrm{X})=0.20 \mathrm{eV}$, which is like that calculated by other PBE exchange potentials, but smaller than the band gap value $0.78 \mathrm{ev}$ obtained by measuring the Holzer coefficient and resistivity, which is caused by the density theory generally underestimates the band gap value.

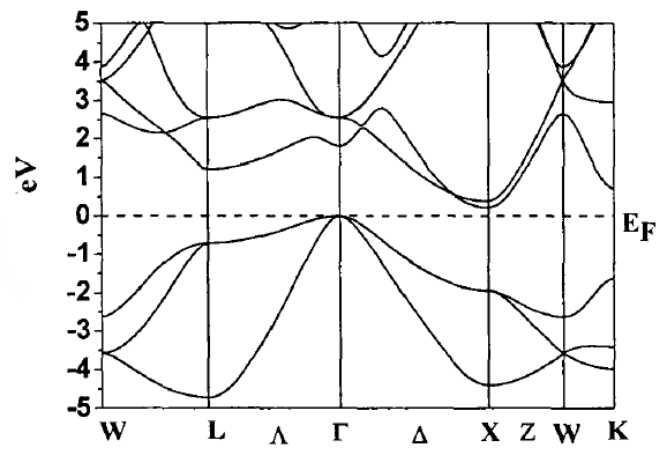

Figure 2. Band structure of Mg2Si

In Figure 3, the partial wave density of states of $\mathrm{Mg}_{2} \mathrm{Si}$ is given. From the chart we can see that the energy in the.9-8eV and clever, the range of $10 \mathrm{ev}, \mathrm{Mg}$ and $\mathrm{Si}$ atoms in orbit have certain hybrid with lower energy range $(-9 \mathrm{eV}--8 \mathrm{eV})$ to $3 \mathrm{~S}$ state electronic density of States is mainly composed of the original Si, Si large electric brake state to state density contribution $151 \mathrm{Oev}$ the scope of the. The electrical transport properties and carrier type of the materials are mainly determined by the electronic structure near the Fermi level. Therefore, the energy density of states near the Fermi level is mainly concerned. It can be seen from the diagram that the energy density of states near the top of the valence band of $\mathrm{Mg}_{2} \mathrm{Si}$ is mainly contributed by the Si state, and the energy density near the conduction band is mainly supplied by the $\mathrm{Mg}$ atom. Based on the eigenvalue of Brillouin region, the transport coefficient is calculated by Boltzmann transport equation. As a thermoelectric material suitable for intermediate temperature region, the variation of transport coefficient with doping concentration is studied. The influence of temperature on the energy band is neglected in the calculation, and the mode and mode doping are simulated by the up and down of the Fermi level.

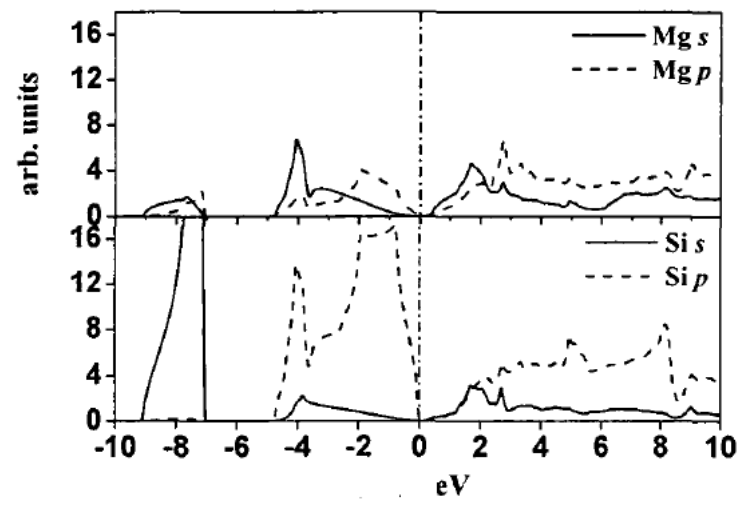

Figure 3. Partial density of states of Mg2Si 


\section{Conclusion}

Si is light element, rich in the earth's crust, and non-toxic, is a harmonious element of the environment. Therefore, if the thermoelectric material with $\mathrm{Si}$ is developed, it will be a low price nontoxic thermoelectric material without rare elements. The relationship between the thermal conductivity of nanocrystalline silicon at room temperature and the thermoelectric value shows that the excellent thermoelectric properties of Nano silicon depend mainly on the reduction of thermal conductivity. Therefore, without reducing the conductivity under the precondition of further reducing the thermal conductivity, the wire structure component is the development direction of silicon in the future.

\section{Acknowledgement}

The paper is the result of Education Quality Enhancement Project of City (State, Region) Regular Undergraduate Colleges of Department of Guizhou Province in 2011 named "Research on the Development of New Environmental High Performance Thermoelectric Materials" (Grant No. 278 of Higher Education Division in Department of Guizhou Province in 2011) led by Shiyun Zhou.

\section{References}

[1] Li Chao, Miao Lei, Liu Chengyan, et al. Review of Nano Si-Ge Thermoelectric Materials and Devices Research [J]. Advances in New And Renewable Energy, 2015, 3(1): 25-32.

[2] Du Ziliang, Chen Shaoping, Wang Yankun. Fabrication and Thermoelectric Performance of the SiNWs-Mg 2 Si Nanocomposite [J]. Rare Metal Materials and Engineering, 2016, 45(10): 2717-2722.

[3] Han Liqin, Yang Meijun, Shen Qiang, et al. Reaction Sintering of Magnesium Silicide Thermoelectric Material by the Spark Plasma Sintering Technique [J]. Journal of the Chinese Ceramic Society, 2008, 36(3): 337-340.

[4] Chen Yuankui, Zhang Zhiwei, Chen Chuan, et al. Synthesis and Thermoelectric Properties of $\mathrm{Zn}$ Doped Oxide Materials $\mathrm{Ca}_{3} \mathrm{Co}_{4} \mathrm{O}_{9}[\mathrm{~J}]$. Journal of Wuhan University of Technology, 2009, 31(4): 25-28. 